\section{Original Article}

\section{Dr. Sultana Amena Ferdoucy}

MBBS, MD

Asst. Professor

Dept. of Radiology and Imaging

Ashiyan Medical College, Khilkhet, Dhaka

Prof. Dr. Md. Anower Hussain Mian BDS, DDS, PhD(Japan)

Post doc. fellow (USA)

Prof. (cc) \& Head Dept. of General

\& Dental Pharmacology

City Dental College \& Hospital

Dr. Nasrin Akhter

M Pharm (DU), Research Fellow (Toxicology) Lecturer

Dept. of Pharmacy

North South University, Dhaka

Dr. Md. Shafiqul Alam

MD (Radiology \& Imaging), MBAS

Clinical Assistant (CA)

Govt. Homeopathic Medical College, Dhaka

Prof. Dr. M. A. Sadek DMRD, FCPS

Dept. of Radiology and Imaging

Bangladesh Institute of Research and Rehabilitation for Diabetes

Endocrine and Metabolic Disorders (BIRDEM),

Dhaka

Correspondence to:

Dr. Sultana Amena Ferdoucy

MBBS, MD

Asst. Professor

Dept. of Radiology and Imaging

Ashiyan Medical College, Khilkhet, Dhaka

E-mail: saferdoucy@yahoo.com

\title{
Correlation between Degenerative Changes and Osteoporosis in Lumbar Spine of Elderly Women in Dhaka city, Bangladesh
}

\begin{abstract}
:
Aims: Degenerative joint diseases and decreased bone mass i.e. osteoporosis are two common age related skeletal disorders responsible for pain and disability. Bangladesh has a high incidence of osteoporosis and the incidence particularly in women, occurs among a relatively younger age group than in the developed world. However little is known about the correlation between degenerative changes and osteoporosis in lumbar spine of elderly women. The purpose of this study was to clarify this relationship in elderly women of Dhaka, Bangladesh.

Methods: A cross-sectional study was conducted at the department of radiology and imaging of Bangladesh institute of research and rehabilitation in diabetes, endocrine and metabolic disorders (BIRDEM), Dhaka during the period of 1st January, 2009 to 31st December, 2010. DEXA scan of spine and BMD measurement were done at a renowned private hospital of Dhaka. Total 63 elderly female aged between $50-75$ years were randomly selected for this study.
\end{abstract}

Results: An inverse relationship between osteoporosis and spondylosis in postmenopausal women as evaluated by bone mineral density and semiquantitative scoring of spinal degeneration was observed. A significant negative correlation $(r=-0.53: p<0.05)$ was found between T-score and grade.

Key words: Spondylosis, osteoporosis, dual energy $\mathrm{x}$-ray absorptiometry (DEXA), bone mineral density (BMD).

\section{Introduction :}

Back pain resulting from degenerative diseases of the spine is one of the most common causes of disability in adults of working age. Between $60 \%$ and $80 \%$ of adults suffer from low back pain at some times in their lives. Back pain results from many causes, including degenerative and congenital spinal stenosis, neoplasm, infection, trauma, and inflammatory or arthritic processes. Acquired spinal stenosis due to degenerative joint and disc diseases accounts for the vast majority of cases. ${ }^{1}$

Osteoarthritis is a degenerative disease can be considered as loss of articular cartilage with accompanying subchondral bone reaction. In primary osteoarthritis, there is no evidence of an initiating predisposing factor. It appears that biomechanical forces across the joint are normal but some intrinsic abnormality of cartilage causes it to degenerate. Secondary osteoarthritis develops from biomechanical imbalance or joints damaged by previous disease such as trauma, inflammatory arthritis, etc. Degenerative spinal diseases are divided according to the site involved. Thus degeneration at the nucleus pulpous leads to disc degeneration (intervertebral osteochondrosis), at the annulus fibroses to spondylosis deformans, at the apophyseal and postvertebral joints to osteoarthritis. 
Degenerative disc disease may occur any where in the spine, but is most common in the lower cervical and lower lumbar. It is characterized by disc space narrowing, reactive sclerosis of adjacent vertebrae, small peripheral osteophytes and intranuclear gas due to the vacuum phenomena. Spondylosis deformans is characterized by osteophytes with a relatively normal or only slightly reduced disc space. Apophyseal joint osteoarthritis occurs predominantly in the cervical and lumbar region and is characterized by joint space narrowing, sclerosis and hypertrophic bone formation. ${ }^{2}$ Coexistence of osteoarthritis and osteoporosis is considered as uncommon. ${ }^{3,9}$ Degenerative disc disease and osteoarthritic changes in the lumbar spine is called lumbar spondylosis. ${ }^{17}$ Lumber disc degeneration is characterized radiologically by the presence of osteophytes, end plate sclerosis and disc space narrowing. ${ }^{4,5}$

Osteoporosis is the most important cause of fracture of the hip, vertebra and distal radius. Osteoporosis has become a health problem in the developed and developing countries. 6 According to World Health Organization (WHO), osteoporosis can be generalized or localized. Again, generalized osteoporosis can be primary and secondary types. Type I primary osteoporosis is osteoclast mediated and is characterized by a rapid bone loss seen in recent postmenopausal women. Therefore, distal radial and vertebral fractures are common. Type II primary osteoporosis is senile, osteoblast mediated and is characterized by age related bone loss, calcium deficiency and /or hyperparathyroidism. Fractures of the proximal femur, especially fracture of the neck of femur and inter-trochanteric fractures are more common in this type of osteoporosis. ${ }^{6}$

Degenerative factors such as, osteophytes, osteochondrosis, scoliosis and vascular calcification are increased from $35 \%$ to $80 \%$ in the 55 to 70 years age group. ${ }^{7}$ It has been accepted generally that conditions of osteoporosis and osteoarthritis are different diseases, possibly resulting from different pathomechanisms. Several investigators have examined the coexistence of osteoporosis and spondylosis in the spine and have reported an inverse relation between decreased bone mineral density (BMD) and intervertebral disc degeneration. . $^{8,10,11}$ Previous studies revealed that an increase in BMD is related with increasing osteophyte and end plate sclerosis grade though not with disc space narrowing. Adjusting for body mass index (BMI) and physical activity does not influence the strength of the association. ${ }^{5}$ Another study suggested that retrolisthesis like other spinal degenerative diseases, was associated with increased spinal BMD. ${ }^{13}$ Osteophytes and end plate sclerosis were more frequent in men than women, but there was no gender difference in the frequency of disc space narrowing. All radiographic features increased in frequency with age; for osteophytes this was true only after excluding mild (grade 1) osteophytes. ${ }^{13}$

The bone density score determines if the patient has osteopenia (low bone mass) or osteoporosis. A woman's bone mass reaches its peak at around the age of 35 years and levels off during menopause. Bone density declines sharply during the first year of menopause. A DEXA scan can detect even a $1 \%$ loss of bone mass. A BMD can be critical to a woman's health later in life when the risk of fracture increases.12 There are several different ways to measure BMD. Dual-energy X-ray absorptiometry (DEXA) is the most accurate way to measure BMD. It uses two different Xray beams to estimate bone density in spine and hip. Strong, dense bones allow less of the X-ray beam to pass through them. The amounts of each X-ray beam that are blocked by bones and soft tissue are compared to each other. ${ }^{14}$ Previous studies reported that an inverse correlation was observed between osteoporosis and spondylosis. ${ }^{9,15}$

Most studies have focused on the association between bone mass and osteoarthritis at the hip, knee, or hand. Less is known about the association between bone mass and degenerative diseases in the spine. However, in the previous studies, only rudimentary qualitative grading systems were used to evaluate the severity of spondylosis. ${ }^{8}$ More quantitative scoring methods for spondylosis appear necessary to achieve more accurate and better determination of the correlation between the bone mass and degeneration. To the best of our knowledge, there is no prior study available in our country to evaluate the possible inverse relationship between osteoporosis and spondylosis. In the current study, the severity of spondylosis was examined using the semi quantitative osteophyte score and the intervertebral disc score from radiographs of the spine. These scores then were examined in relation to the BMD measurements at the lumbar spine.

\section{Materials \& Methods:}

\section{Selection of patients:}

Inclusion criteria-

1. Elderly female subjects (50-75 years).

2. Spinal radiograph and DEXA scan of spine were done.

\section{Exclusion criteria-}

1. Subjects under hormone replacement therapy.

2. Interval between X-ray spine and DEXA scan was more than 1 year.

3 . History of hysterectomy.

Sample size: Estimated final sample size was 63. 


\section{Methods:}

The patients who were sent for X-ray lumbar spine in department of radiology and imaging and fulfill the inclusion criteria included in this study. Lumbar spine radiographs were taken according to a standard protocol with the film centered at L3 vertebra. Anteroposterior and lateral views were taken. The radiographs were evaluated by using a semi quantitative scoring system of Kellgren and Lawrence score. ${ }^{16}$

\section{Kellgren and Lawrence score:}

Grade-I: Minimal osteophytosis only.

Grade-II: Definite osteophytosis with some sclerosis of anterior part of vertebral plates.

Grade-III: Marked osteophytosis and sclerosis of vertebral plates and marked narrowing of disk space.

Grade-IV: Large osteophytosis, marked sclerosis of vertebral plates and marked narrowing of disk space. Osteoporosis was measured by DEXA scan by using T-scores. The WHO uses T-scores ${ }^{18}$ to define normal bone mass, low bone mass (or osteopenia), and osteoporosis. T-score compares bone density to the average bone density of young healthy adults of same gender.

Normal $=$ T-score $\geq 1$.

Osteopenia $=\mathrm{T}$-score between -1 and -2.5 .

Osteoporosis $=\mathrm{T}$-score $\leq-2.5$.

A sum-score used to correlate the findings with the BMD.

\section{Results:}

The severity of spondylosis was examined using the semi quantitative osteophyte score and the intervertebral disc score from radiographs of the spine. These scores then examined in relation to the bone mineral density (BMD) measurements at the lumbar spine. BMD measurements were evaluated by DEXA scan using T score.

The mean age of the patient was 66.5 years and ranged from 50 to 75 years. They were divided into 3 groups. The highest incidence of back pain was found in the age group between $61-70$ years. The mean Tscore test was -1.7 with standard error of mean $( \pm S E)$ \pm 0.16 with T-score test ranged from -5 to 2 . Majority $(47.6 \%)$ of the patients had osteopenia, $28.6 \%$ had osteoporosis and rest $23.8 \%$ was found normal according to T-score test. Maximum (74.6\%) of the study patients suffered from grade II type of osteoporosis, and then $12.7 \%$ grade III, $9.5 \%$ grade I and $3.2 \%$ from grade IV type of osteoporosis. A significant negative correlation $(r=-0.531 ; p<0.05)$ was found between T-score and grade.
Table-1: Distribution of study subjects according to age group. ( $n=63)$

\begin{tabular}{lcc}
\hline Age group & $\mathrm{n}$ & $\%$ \\
\hline $50-60$ & 4 & 6.3 \\
$61-70$ & 44 & 69.8 \\
$>70$ & 15 & 23.8 \\
\hline MeantSE & \multicolumn{2}{c}{$66.5 \pm 1.24$} \\
Range & \multicolumn{2}{c}{$50-75$ years } \\
\hline
\end{tabular}

Age ranged from 50 to 75 years and the maximum number was found in the age group of 61-70 years. The mean age was 66.5 years with standard error of mean $( \pm S E) \pm 1.24$ years. The results are shown in the table-1. The study included 63 elderly female patients having back pain. Spinal radiograph and DEXA scan of spine were done in all 63 cases. They were divided into three age groups.

Table-2: Distribution of study subjects according to their T-score test. ( $n=63)$

\begin{tabular}{lcc}
\hline & $n$ & $\%$ \\
\hline Normal & 15 & 23.8 \\
Osteopenia & 30 & 47.6 \\
Osteoporosis & 18 & 28.6 \\
\hline MeantSE & \multicolumn{3}{c}{$-1.7 \pm 0.16$} \\
Range & \multicolumn{3}{c}{$(-5-2)$} \\
\hline
\end{tabular}

Highest number (47.6\%) of the patients had osteopenia followed by osteoporosis $(28.6 \%)$ and the rest $(23.8 \%)$ were found normal according to T-score test. The mean T-score test was -1.7 with standard error of mean $( \pm \mathrm{SE}) \pm 0.16$ with T-score test ranged from -5 to 2 (table-2).

Table-3: Distribution of study subjects according to Kellgren and Lawrence score.

\begin{tabular}{lcc} 
& & (n=63) \\
\hline Grade I & $n$ & $\%$ \\
Grade II & 6 & 9.5 \\
Grade III & 47 & 74.6 \\
Grade IV & 8 & 12.7 \\
\hline
\end{tabular}

Table-3 shows the distribution of the study patients according to Kellgren and Lawrence score to determine the presence of degenerative changes of each lumbar vertebra. The patients were divided into four grades using Kellgren and Lawrence score. Majority (74.6\%) of the study patients suffered from grade II type of osteoporosis and then $12.7 \%$ from grade III, $9.5 \%$ grade I and $3.2 \%$ grade IV type. 


\section{Graph-1: Correlation between T-score and Grade of the study subjects. $(n=63)$}

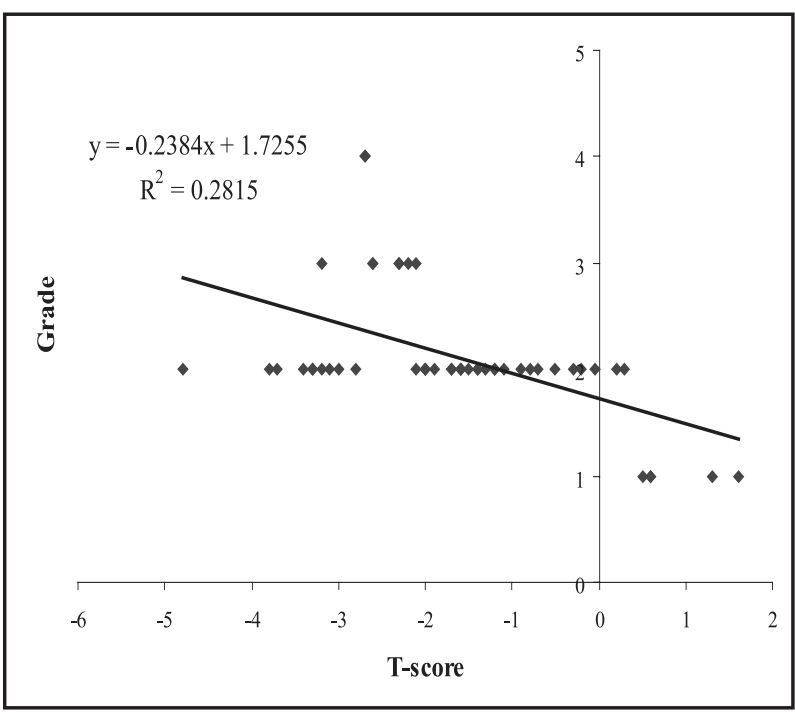

The scatter diagram shows significant negative relationship ( $r=-0.531)$ between T-score and grade. The values of Pearson's correlation coefficient were $r=-0.531$ which was significant $(p<0.05)$.

\section{Discussion:}

Between $60 \%$ and $80 \%$ of adults suffer from low back pain at some times in their lives.1 Back pain results from many causes, including degenerative and congenital spinal stenosis, neoplasm, infection, trauma, and inflammatory or arthritic processes. 1 Degenerative factors such as, osteophytes, osteochondrosis, scoliosis and vascular calcification are increased from $35 \%$ to $80 \%$ in the 55 to 70 years age group.7 Elderly women of 50-75 (mean age of $66.5)$ years were included in this study (table-1). The severity of spondylosis was evaluated through spinal radiographs using a semi quantitative scoring system of Kellgren and Lawrence score16 (table-3). Presence of degenerative changes (osteoporosis) by means of bone mineral density (BMD) of each lumbar vertebra was measured by DEXA scan expressed as T-scores (table-2). The scatter diagram showed a significant $(p<0.05)$ negative relationship $(r=-0.531)$ between Tscore and grade (fig.1). Therefore, a linear negative correlation was observed between T-score and grade.

\section{Conclusions:}

In the present study, relationship between bone mineral density in lumbar spine and severity of spondylosis was evaluated in elderly females. On the basis of findings of the study it can be concluded that osteoporosis has an inverse relationship with spondylosis. However, further study can be carried out by taking larger sample size using MRI, CT scan and bone mineral density of remote site in addition to lumber spine.

\section{References:}

1. David SJ (editor). In: John RH, Charles FL. CT and MR Imaging of the whole body. 4th ed. United States of America:2003;2:724-64.

2. William A, Murphy J, Bryan JP (editor). In: Grainger RG, Allison DJ, Dixon AK. Grainger and Allison's Diagnostic Radiology. 4th ed. Churchill Livingstone; Adama:2003;3:1995-2026.

3. Pye SR, Reid DM, Adams R, et al. Radiographic features of lumbar disc degenerative-reported back pain. J Rheumatol 2004;31:753-58.

4. Stephen RP, David MR, Mark L, et al. Lumbar disc degeneration: association between osteophytes, endplate sclerosis and disc space narrowing. Ann Rheum Dis 2007;66:330-33.

5. Pye SR, Reid DM, Admas JE, et al. Two radiographic features of lumbar disc degeneration and bone mineral density in men and women. Ann Rheum Dis 2006;65:234-38.

6. Bale A (editor). In: Osteoporosis. 1st ed. New Delhi, India: The South East Asia Regional office, WHO;2000:1-18.

7. Rand T, Sedil G, Kainberger F, et al. Impact of spinal degenerative changes on evaluation of bone mineral density with duel energy Xray absorptiometry. Calcif Tissue Int 1997;60(5):430-33.

8. Miyakoshi $\mathrm{N}$, Itoi $\mathrm{E}$, Murai $\mathrm{H}$, et al. Inverse relation between osteoporosis and spondylosis in postmenopausal women as evaluated by bone mineral density and semiquantitative scoring of spinal degeneration. Spine 2003;28:492-95.

9. Hart DJ, Mootoosamy I, Doyle DV, et al. The relationship between osteoarthritis and osteoporosis in the population: the Chingfords. Ann Rheum Dis 1994;53:158-62.

10.Nuti R, Righi G, Marini G, et al. Diagnostic approach to osteoporosis and spondyloarthrosis in postmenopausal women by total body dual photon absorptiometry. Clin Exp Rheum 1998;6:47-51.

11. Roux C, Fechtenbaum J, Briot K, et al. Inverse relation between vertebral fracture and spine osteoarthitis in postmenopausal women with osteoporosis. Ann Rheum Dis 2008; 67: 224-28.

12.Spinasanta S. Bone mineral density (BMD) measurement. www.spineuniverse.com/displayarticle. php/article264.heml

13. Vogt MT, Rubin DA, sanValentin $R$, et al. Degenerative lumbar listless and bone mineral density in elderly women. The study of osteoporotic spine 1999;24(23):2536-41.

14. Osteoporosis Health Center. Bone Mineral Density. July 2009. www.webmd.com/osteoporosis/bone-mineral-density

15. Harada A, Okuizumi H, Miyagi N, et al. Correlation between bone mineral density and intervertebral disc degeneration. Spine 1998;23(8)857-61.

16. Schmitt H, Friebe C, Schneider S, et al. Bone density and degenerative changes of the lumbar spine in former elite athletes. Int j Sports Med 2005;26:463.

17.Doherty M, Ralston SH. In: Davidson's principles and practice of medicine. 21st ed. Churchill Livingstone: London; 2010:1145

18. Reach A, Schneider B, Batman A, et al. Risk of vertebral fractures in men: relationship to mineral density of the vertebral body, American J Roentgentol 1995;164:224. 